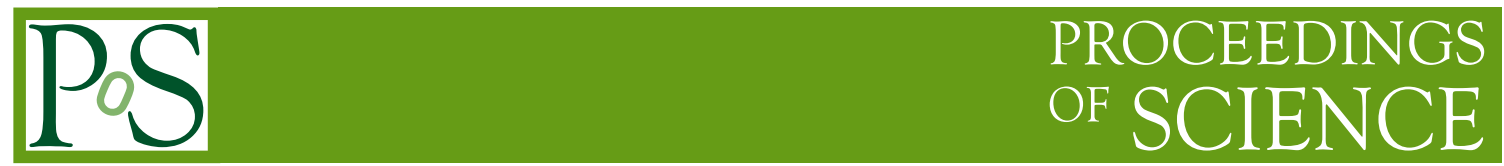

\title{
Study of QCD in gamma gamma to pseudoscalar meson pair processes
}

Hideyuki Nakazawa (Belle Collaboration)

National Central University (R.O.C.)

E-mail: nkzwepost.kek.jp

We have measured a series of exclusive meson-pair productions in two-photon collision at the Belle experiment. Above around $3 \mathrm{GeV}$ of two-photon invariant mass, the measured cross sections and angular distributions are compared with perturbative and non-perturbative QCD calculations.

36th International Conference on High Energy Physics,

July 4-11, 2012

Melbourne, Australia 

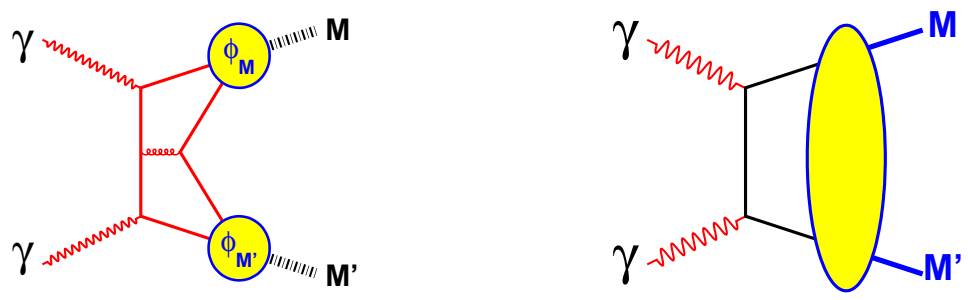

Figure 1: Factorization of the process $\gamma \gamma \rightarrow M M^{\prime}$ by perturbative QCD (left) and Handbag picture (right).

Exclusive meson-pair production in two-photon collision, $\gamma \gamma \rightarrow M M^{\prime}$ provides useful information for study of perturbative and non-perturbative QCD. From theoretical viewpoint, two-photon process is attractive because of the absence of strong interactions in the initial state and the possibility of calculating $\gamma \gamma \rightarrow q \bar{q}$ amplitudes.

Brodsky and Lepage (BL) [四] have computed the amplitude for the $\gamma \gamma \rightarrow M M^{\prime}$ process for the first time (Fig.W left). Their perturbative QCD calculation is obtained by factorizing the amplitude into two components,

$$
\mathscr{M}_{\lambda_{1} \lambda_{2}}\left(s, \theta^{*}\right)=\int_{0}^{1} \int_{0}^{1} d x d y \phi_{M}\left(x, Q_{x}\right) \phi_{M^{\prime}}\left(y, Q_{y}\right) T_{\lambda_{1} \lambda_{2}}\left(x, y, \theta^{*}\right),
$$

where $\phi_{M}\left(x, Q_{x}\right)$ is a single-meson distribution amplitude for a meson $M$, the probability amplitude for finding valence partons in the meson, each carrying some fraction $x$ of the meson's momentum. $Q_{x}$ is the typical momentum transfer in the process, $\sim \min (x, 1-x) \sqrt{s}\left|\sin \theta^{*}\right|$ with meson scattering angle $\theta^{*}$ in the two-photon c.m.s. By the sum rule the overall normalization is fixed as $\int_{0}^{1} d x \phi_{M}\left(x, Q_{x}\right)=f_{M} / 2 \sqrt{3}$ where $f_{M}$ is the decay constant for a meson $M . T_{\lambda_{1}} \lambda_{2}$ is a hard scattering amplitude for $\gamma_{\lambda_{1}} \gamma_{\lambda_{2}} \rightarrow q \bar{q} q \bar{q}$ with photon helicities $\lambda_{1}$ and $\lambda_{2}$.

For mesons with zero helicity leading term calculation gives the following dependence on $s$ and scattering angle $\theta^{*}$ :

$$
\frac{d \sigma}{d\left|\cos \theta^{*}\right|}=16 \pi \alpha^{2} \frac{\left|F_{M}(s)\right|^{2}}{s}\left\{\frac{\left[\left(e_{1}-e_{2}\right)^{2}\right]^{2}}{\left(1-\cos ^{2} \theta^{*}\right)^{2}}+\frac{2\left(e_{1} e_{2}\right)\left[\left(e_{1}-e_{2}\right)^{2}\right]}{1-\cos ^{2} \theta^{*}} g\left(\theta^{*}\right)+2\left(e_{1} e_{2}\right)^{2} g^{2}\left(\theta^{*}\right)\right\}
$$

where $e_{1}$ and $e_{2}$ are the quark charges. Under the assumption that $\phi_{K}$ and $\phi_{\pi}$ are similar in shape, the differential cross section ratio depends only on the meson decay constants $f_{K}^{4} / f_{\pi}^{4}$ for the charged mode. Benayoun and Chernyak (BC) [వ] employ different wave functions for $\phi_{\pi}(x)$ and $\phi_{K}(x)$ taking into account SU(3) symmetry breaking effects. Next-to-leading order calculation is done by Duplančić et al. [[]].

The Handbag model by Diehl, Kroll and Vogt (DKV) [四] predicted the differential cross section for the $\gamma \gamma \rightarrow M M^{\prime}$ process as

$$
\frac{d \sigma}{d\left|\cos \theta^{*}\right|}\left(\gamma \gamma \rightarrow M M^{\prime}\right)=\frac{8 \pi \alpha^{2}}{s} \frac{1}{\sin ^{4} \theta^{*}}\left|R_{M M^{\prime}}(s)\right|^{2},
$$

where the transition amplitude is expressed as a hard scattering $\gamma \gamma \rightarrow q \bar{q}$ times a form factor

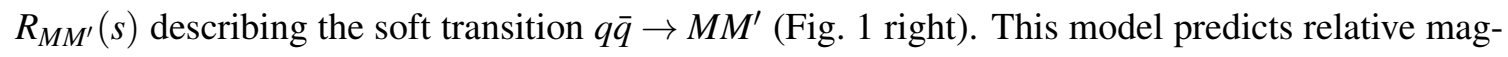
nitude of the cross sections between various modes, while it does not give absolute magnitude of the cross section. 

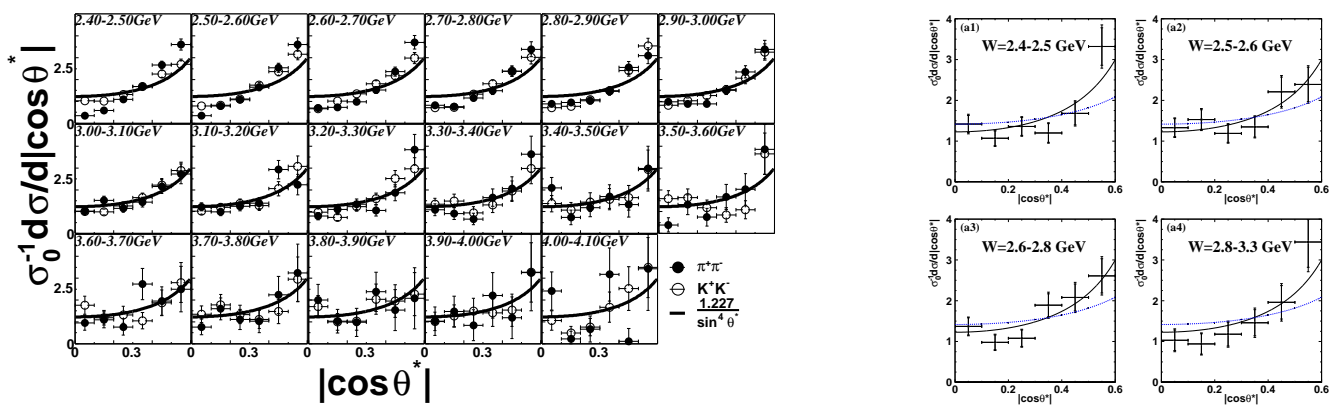

Figure 2: Angular dependence of the normalized differential cross section for $\gamma \gamma \rightarrow K^{+} K^{-}$and $\pi^{+} \pi^{-}$(left) and $K_{S} K_{S}$ (right). Solid curves are $\sin ^{-4} \theta^{*}$ dependence. Blue curves show prediction by BC.

(a)
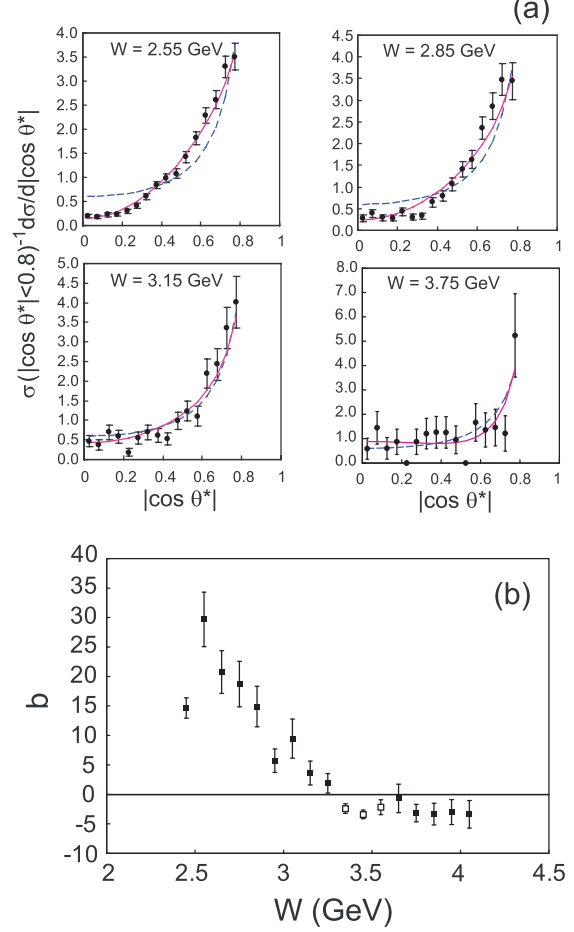

(c)

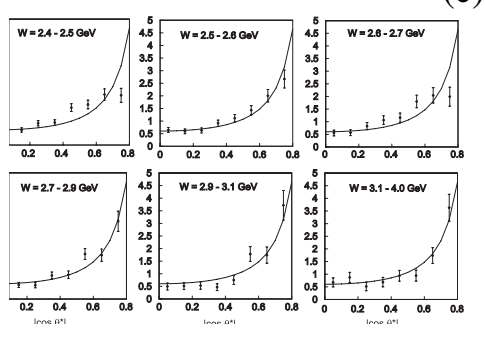

(d)

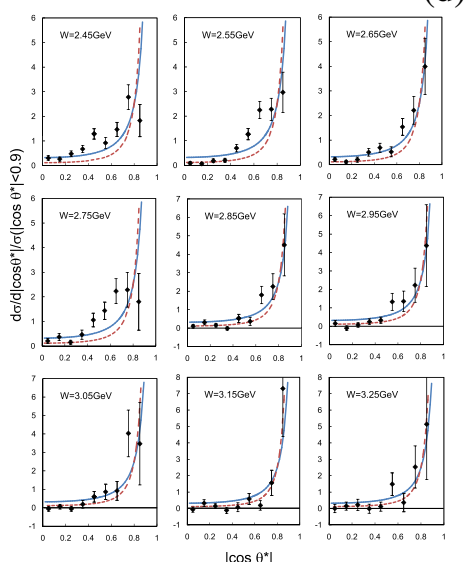

Figure 3: Angular dependence of the normalized differential cross section: (a) $\gamma \gamma \rightarrow \pi^{0} \pi^{0}$, (c) $\eta \pi^{0}$, and (d) $\eta \eta$. (a): Dotted (solid) curves show $\sin ^{-4} \theta^{*}$ (fit to $\sin ^{-4} \theta^{*}+b \cos \theta^{*}$ ) dependence. $W$ dependence of $b$ is shown in (b). (c): Curves show $\sin ^{-4} \theta^{*}$. (d): Dotted (solid) curves show $\sin ^{-6} \theta^{*}\left(\sin ^{-4} \theta^{*}\right)$ dependence.

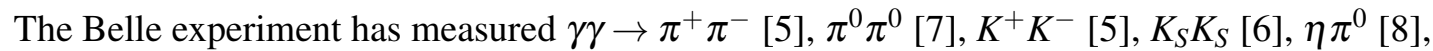
and $\eta \eta$ [Q] processes. The results are compared with perturbative and non perturbative QCD predictions.

\section{Angular Dependence of Differential Cross Section}

In Equation (DI) the first term is dominant for charged pair mode, and the angular distribution is thus expected to have $\sim \sin ^{-4} \theta^{*}$ dependence. But for neutral pair mode for which the first term vanishes the angular dependence is directly determined by the shape of $g\left(\theta^{*}\right)$ and the value of 
Table 1: Angular dependence of differential cross sections in comparison with $\sin ^{-4} \theta^{*}$ dependence.

\begin{tabular}{|c|c|c|c|c|}
\hline mode & $\sin ^{-4} \theta^{*}$ & energy range & $\left|\cos \theta^{*}\right|$ range & reference \\
\hline$\pi^{+} \pi^{-}$ & Match well. & $3.0-4.1$ & $<0.6$ & [5] \\
\hline$K^{+} K^{-}$ & Match well. & $3.0-4.1$ & $<0.6$ & [5] \\
\hline$K_{S} K_{S}$ & Consistent. & $2.4-3.3$ & $<0.6$ & [焑] \\
\hline$\pi^{0} \pi^{0}$ & $\begin{array}{l}\sin ^{-4} \theta^{*}+b \cos \theta^{*} \text { better. } \\
\text { Approaches } \sin ^{-4} \theta^{*} \text { above } 3.1 \mathrm{GeV}\end{array}$ & $2.4-4.1^{\dagger}$ & $<0.8$ & [四] \\
\hline$\eta \pi^{0}$ & Good agreement above $2.7 \mathrm{GeV}$. & $3.1-4.1$ & $<0.8$ & [8] \\
\hline$\eta \eta$ & $\begin{array}{l}\text { Poor agreement. } \\
\sin ^{-6} \theta^{*} \text { better above } 3.0 \mathrm{GeV}\end{array}$ & $2.4-3.3$ & $<0.9$ & [9] \\
\hline
\end{tabular}

$F_{M}(s)$, which depend on incalculable factor $\phi_{M}$. On the other hand, the handbag model predicts $\sin ^{-4} \theta^{*}$ dependence for large $t$ both for charged and neutral meson pairs.

The measured angular dependence are consistent with $\sin ^{-4} \theta^{*}$ around $3 \mathrm{GeV}$ or higher energy region except $\eta \eta$ mode. Figure $\square$ shows the measured angular dependence for $\pi^{+} \pi^{-}, K^{+} K^{-}$, and $K_{S} K_{S}$. For $\gamma \gamma \rightarrow \pi^{0} \pi^{0}$ and $\eta \eta, \sin ^{-4} \theta^{*}+b \cos \theta^{*}$ and $\sin ^{-6} \theta^{*}$ dependence, respectively, show better agreement than $\sin ^{-4}$ while $\eta \pi^{0}$ is in agreement with $\sin ^{-4} \theta^{*}$ above $2.7 \mathrm{GeV}$ (Fig. [1]). Comparison with $\sin ^{-4} \theta^{*}$ dependence is summarized in Table $\mathbb{W}$.

\section{Energy Dependence of Cross Section and ratio of Cross Sections}

It is found that existing calculations do not agree with absolute normalization of the cross sections even with next-to-leading-order term [B]. However, power-low dependence of cross section $\sigma_{0} \sim W^{-n}$ and their ratio, summarized in Table $\square$, provide useful information to test QCD predictions.

Figure $\mathbf{G}$ (Figure (1) shows cross sections integrated over sensitive angular region for $\gamma \gamma \rightarrow$ $K_{S} K_{S}$ and $\pi^{0} \pi^{0}\left(\eta \pi^{0}\right.$ and $\left.\eta \eta\right)$ and their ratios to charged $\left(\pi^{0} \pi^{0}\right)$ mode. The range of all measured $n$ value, from 7 to 10 , is not far above the asymptotic pQCD prediction of 6 [ए]]. At present energies, the leading term may be small and dominated by the first power correction, therefore energy dependence can be much steeper, $n \sim 10$ []]. Cross section ratio, $\sigma_{0}\left(K^{+} K^{-}\right) / \sigma_{0}\left(\pi^{+} \pi^{-}\right)$ is constant in present energy region, while neutral-to-charged ratios, $\sigma_{0}\left(K_{S} K_{S}\right) / \sigma_{0}\left(K^{+} K^{-}\right)$and $\sigma_{0}\left(\pi^{0} \pi^{0}\right) / \sigma_{0}\left(\pi^{+} \pi^{-}\right)$seem to approach constant. Cross sections for $\eta \pi^{0}, \eta \eta, K^{+} K^{-}$, and $K_{S} K_{S}$

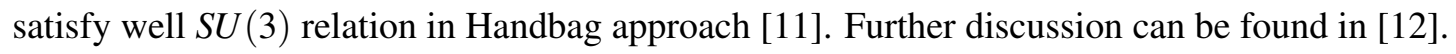

\section{References}

[1] S. J. Brodsky and G. P. Lepage, Phys. Rev. D 24, 1808 (1981).

[2] M. Benayoun and V. L. Chernyak, Nucl. Phys. B 329, 285 (1990).

[3] G. Duplančić et al., Phys. Lev. Lett 97, 142003 (2006). 

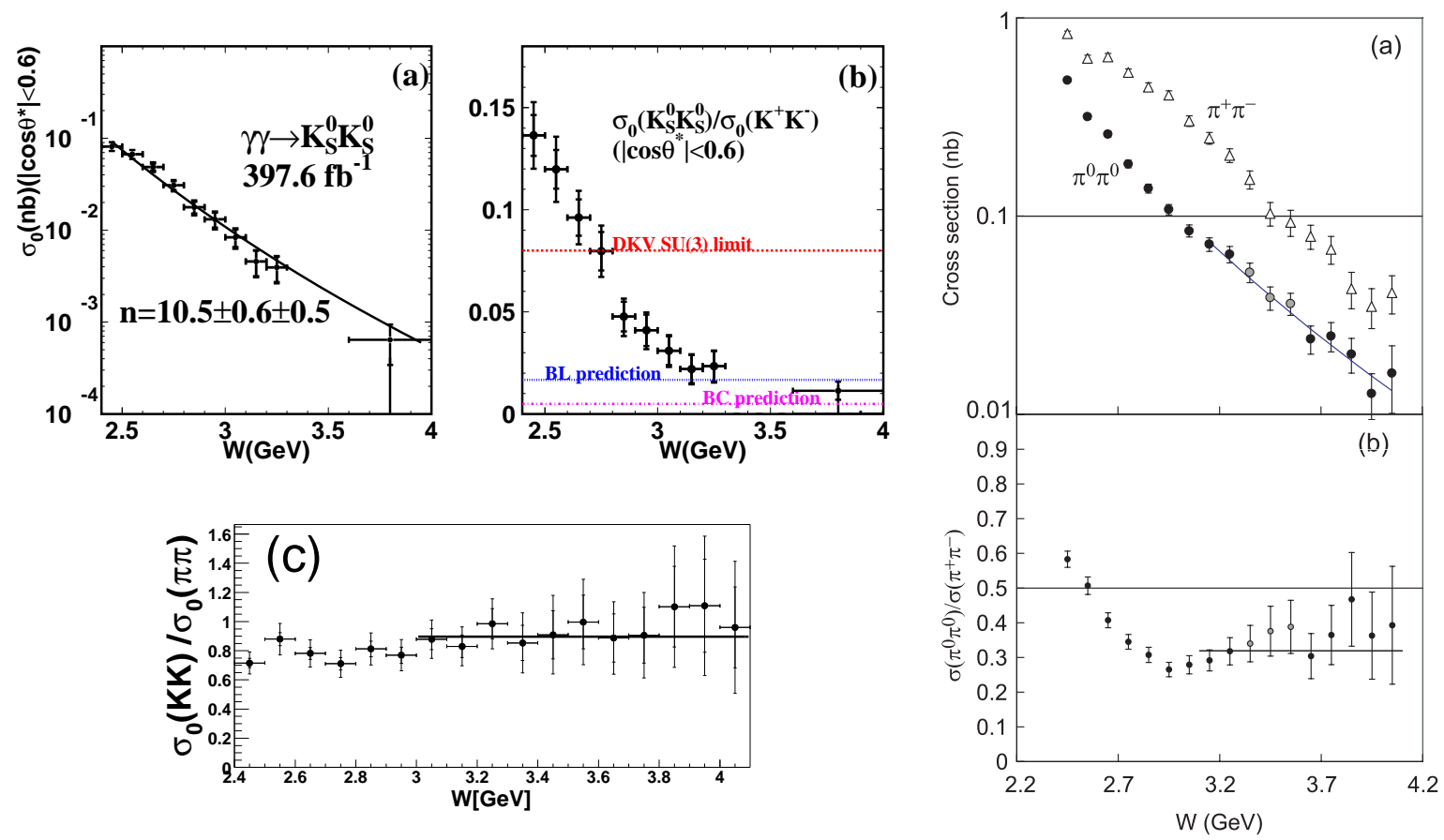

Figure 4: Left: (a) Cross section for $\gamma \gamma \rightarrow K_{S} K_{S}$, (b) its ratio to $K^{+} K^{-}, \sigma_{0}\left(K_{S} K_{S}\right) / \sigma_{0}\left(K^{+} K^{-}\right)$ and (c) $\sigma_{0}\left(K^{+} K^{-}\right) / \sigma_{0}\left(\pi^{+} \pi^{-}\right)$. Right: (a) Cross section for $\pi^{0} \pi^{0}$ and $\pi^{+} \pi^{-}$and (b) their ratio $\sigma_{0}\left(\pi^{0} \pi^{0}\right) / \sigma_{0}\left(\pi^{+} \pi^{-}\right)$. Curves in (a) show fits to $W^{-n}$. Fit result to a constant value is shown for $\sigma_{0}\left(K^{+} K^{-}\right) / \sigma_{0}\left(\pi^{+} \pi^{-}\right)$and $\sigma_{0}\left(\pi^{0} \pi^{0}\right) / \sigma_{0}\left(\pi^{+} \pi^{-}\right)$.
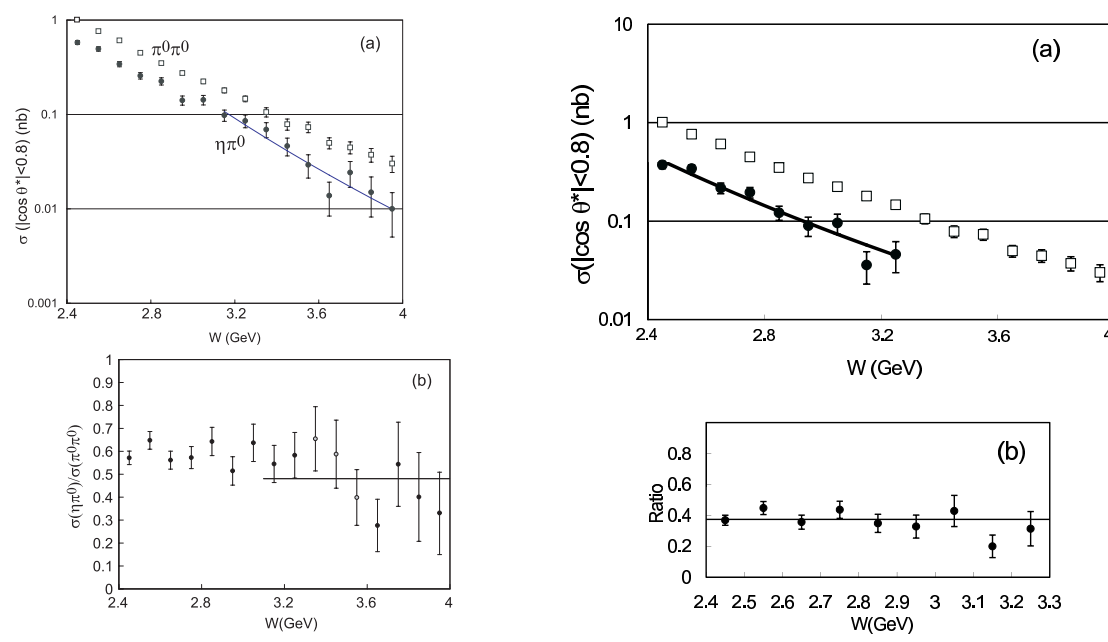

Figure 5: Cross section and its ratio to $\pi^{0} \pi^{0}$ for $\eta \pi^{0}$ (left) and $\eta \eta$ (right). In (a), $\sigma_{0}\left(\pi^{0} \pi^{0}\right)$ is shown in open squares. Curves are fit result to $W^{-n}$ dependence. 
Table 2: The value of $n$ of $\sigma_{0} \propto W^{-n}$ in various reactions fitted in the $W$ and $\left|\cos \theta^{*}\right|$ ranges indicated and the ratio of the cross sections in comparison with QCD predictions. The first and second errors are statistical and systematic, respectively.

\begin{tabular}{lcccccc}
\hline \hline Process & $n$ or $\sigma_{0}$ ratio & $W(\mathrm{GeV})$ & $\left|\cos \theta^{*}\right|$ & BL [四] & BC [వ] & DKV [田] \\
\hline$\pi^{+} \pi^{-}$ & $7.9 \pm 0.4 \pm 1.5$ & $3.0-4.1$ & $<0.6$ & 6 & 6 & \\
$K^{+} K^{-}$ & $7.3 \pm 0.3 \pm 1.5$ & $3.0-4.1$ & $<0.6$ & 6 & 6 & \\
$K_{S}^{0} K_{S}^{0}$ & $10.5 \pm 0.6 \pm 0.5$ & $2.4-4.0^{1}$ & $<0.6$ & 6 & 10 & \\
$\pi^{0} \pi^{0}$ & $8.0 \pm 0.5 \pm 0.4$ & $3.1-4.1^{\dagger}$ & $<0.8$ & 6 & 10 & \\
$\eta \pi^{0}$ & $10.5 \pm 1.2 \pm 0.5$ & $3.1-4.1$ & $<0.8$ & 6 & 10 & \\
$\eta \eta$ & $7.8 \pm 0.6 \pm 0.4$ & $2.4-3.3$ & $<0.8$ & 6 & 10 & \\
\hline$K^{+} K^{-} / \pi^{+} \pi^{-}$ & $0.89 \pm 0.04 \pm 0.15$ & $3.0-4.1$ & $<0.6$ & 2.3 & 1.06 & \\
$K_{S} K_{S} / K^{+} K^{-}$ & $\sim 0.13$ to $\sim 0.01$ & $2.4-4.0$ & $<0.6$ & & 0.005 & $2 / 25$ \\
$\pi^{0} \pi^{0} / \pi^{+} \pi^{-}$ & $0.32 \pm 0.03 \pm 0.06$ & $3.1-4.1$ & $<0.6$ & & $0.04-0.07$ & 0.5 \\
$\eta \pi^{0} / \pi^{0} \pi^{0}$ & $0.48 \pm 0.05 \pm 0.04$ & $3.1-4.0$ & $<0.8$ & $0.24 R_{f}\left(0.46 R_{f}\right)^{\ddagger}$ & & \\
$\eta \eta / \pi^{0} \pi^{0}$ & $0.37 \pm 0.02 \pm 0.03$ & $2.4-3.3$ & $<0.8$ & $0.36 R_{f}^{2}\left(0.62 R_{f}^{2}\right)^{\ddagger}$ & & \\
\hline \hline \multicolumn{7}{c}{$\dagger \chi_{c J}$ region, 3.3-3.6 GeV is excluded. }
\end{tabular}

$\ddagger \eta$ meson as a pure $\mathrm{SU}(3)$ octet (mixture of octet and singlet with $\theta_{p}=-18^{\circ}$ ), $R_{f}=f_{\eta}^{2} / f_{\pi^{0}}^{2}$.

[4] M. Diehl, P. Kroll and C. Vogt, Phys. Lett. B 532, 99 (2002).

[5] H. Nakazawa et al. (Belle Collaboration), Phys. Lett. B 615, 39 (2005).

[6] W.T. Chen et al. (Belle Collaboration), Phys. Lett. B 651, 15 (2007).

[7] S. Uehara et al. (Belle Collaboration), Phys. Rev. D 79, 052009 (2009).

[8] S. Uehara et al. (Belle Collaboration), Phys. Rev. D 80, 032001 (2009).

[9] S. Uehara et al. (Belle Collaboration), Phys. Rev. D 82, 114031 (2010).

[10] S. J. Brodsky and G. R. Farrar, Phys. Rev. D 11, 1309 (1975).

[11] M. Diehl and P. Kroll, Phys. Lett. B 683, 165 (2010).

[12] V.L. Chernyak, Phys. Lett. B 640 (2006) 246. 